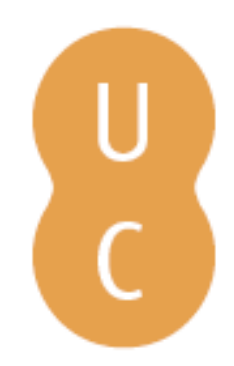

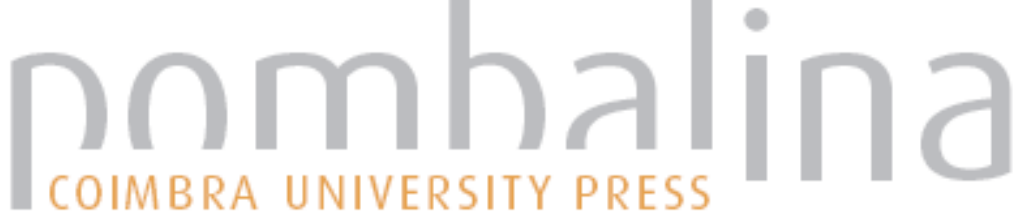

Un nomos atopos? Gli Efori e i baffi degli Spartani: nota esege tica a De sera num. vind. 4.550B
Autor(es):
Amendola, Stefano
Publicado por: Imprensa da Universidade de Coimbra
URL
persistente:
URI:http://hdl.handle.net/10316.2/32878
DOI:
DOI:http://dx.doi.org/10.14195/978-989-721-012-9_9
Accessed : $\quad$ 26-Apr-2023 11:16:47

A navegação consulta e descarregamento dos títulos inseridos nas Bibliotecas Digitais UC Digitalis, UC Pombalina e UC Impactum, pressupõem a aceitação plena e sem reservas dos Termos e Condições de Uso destas Bibliotecas Digitais, disponíveis em https://digitalis.uc.pt/pt-pt/termos.

Conforme exposto nos referidos Termos e Condições de Uso, o descarregamento de títulos de acesso restrito requer uma licença válida de autorização devendo o utilizador aceder ao(s) documento(s) a partir de um endereço de IP da instituição detentora da supramencionada licença.

Ao utilizador é apenas permitido o descarregamento para uso pessoal, pelo que o emprego do(s) título(s) descarregado(s) para outro fim, designadamente comercial, carece de autorização do respetivo autor ou editor da obra.

Na medida em que todas as obras da UC Digitalis se encontram protegidas pelo Código do Direito de Autor e Direitos Conexos e demais legislação aplicável, toda a cópia, parcial ou total, deste documento, nos casos em que é legalmente admitida, deverá conter ou fazer-se acompanhar por este aviso. 


\section{Nomos, Kosmos \& Dike in Plutarch}

José Ribeiro Ferreira, Delfim F. Leão \& Carlos A. Martins de Jesus (eds.) 


\title{
Un nomos atopos? Gli Efori e i bafFi degli Spartani. Nota ESEGETICA A DE SERA NUM. VIND. 4.550B
}

Stefano Amendola

Università di Salerno

\begin{abstract}
At De sera num. vind. 550B, among the examples of atypical laws, Plutarch cites the ban issued by the Spartan Ephors to let the whiskers grow. Witness of this prescription is Aristotle (fr. 539 Rose), specifically mentioned by Plutarch at Cleom. 9.3, where the latter again refers to the odd Spartan law: the text of the biography, however, shows how the same nomos, held as atopos in the aforementioned passage of the treatise, has indeed a precise motivation for Plutarch. Here we propose to discuss some of the ecdotic and exegetic doubts raised by a comparison between the two texts, and further consider the third witness of the Aristotelian quote, Plu. fr. 90 Sandbach.
\end{abstract}

Nel quarto capitolo del De sera numinis vindicta (549D-550C) Plutarco dà inizio al suo primo discorso: l'intervento del Cheronese è presentato come necessario ad evitare che ulteriori dubbi, posti dagli altri protagonisti del dialogo, rafforzino a tal punto le critiche mosse dall'anonimo epicureo contro la provvidenza divina da renderne impossibile la confutazione (549E). Lintero capitolo appare incentrato sulla polarità umano/ divino: in particolare, il Cheronese sembrerebbe proporre una sorta di paragone tra alcune technai umane e quella che Plutarco chiama $\pi \alpha \sigma \tilde{\omega} v$

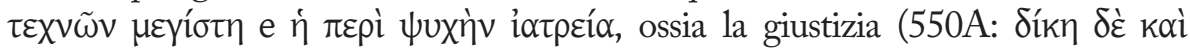

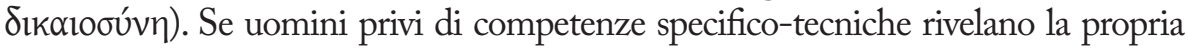
ignoranza intervenendo in campi 'propriamente umani', quali musica, strategia bellica e medicina, un mortale, proprio in quanto mortale, non può comprendere l'operato della divinità, i tempi e i modi con i quali castiga i malvagi, essendo sprovvisto di

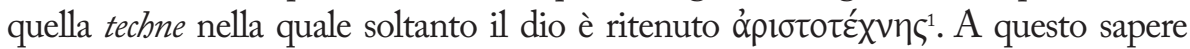
divino è stato invece educato Minosse, figlio e allievo di Zeus: relativamente alla figura del re cretese Plutarco rimanda a Platone' , il quale a sua volta recupera dai poeti epici (Omero ed Esiodo) un'immagine totalmente positiva del sovrano, ben lontana da quella violenta e barbarica offerta dai tragici. Il Cheronese fa riferimento a $\mathrm{Pl}$. $L g$. 614a-b e, in particolare, a [Pl.] Min. 318e-319f: in quest'ultimo dialogo, a partire

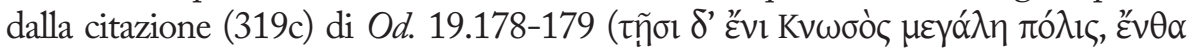

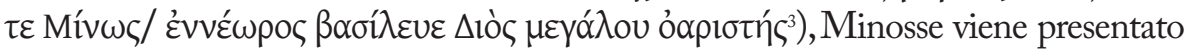

\footnotetext{
${ }^{1}$ Su valore e funzione del termine cf. F. Frazier: 219 in questo stesso volume.

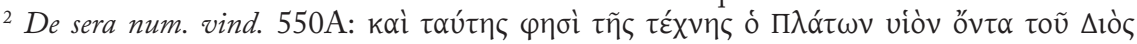

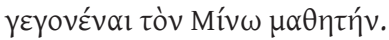

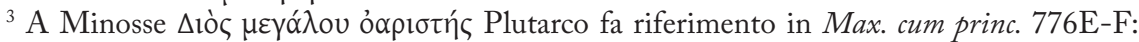

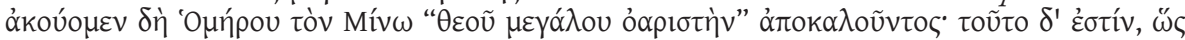

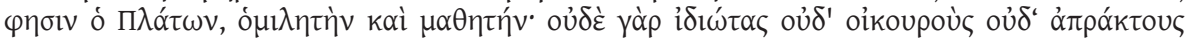


come sovrano in costante contatto con la divinità, educato direttamente dal padre

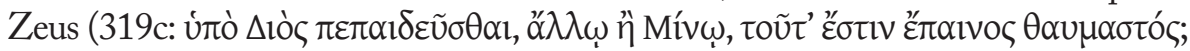

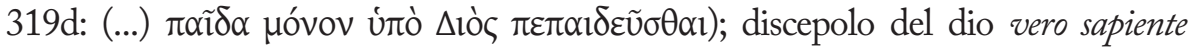

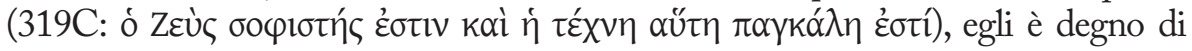
essere rappresentato nella Nekyia odissiaca $(O d .11 .568-571)$ quale giudice infernale

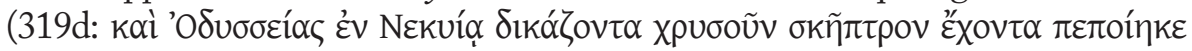
tòv Mív $\omega v)$. In netto contrasto con la citata figura di Minosse, esempio di regnante e legislatore semidivino ${ }^{4}$, Plutarco torna ad evidenziare l'aporia dei mortali che, non solo non sono capaci di comprendere le forme della giustizia divina, ma ignorano anche la ragione di alcune leggi umane, che finiscono per apparire strane e finanche

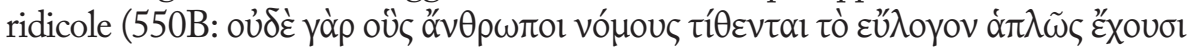

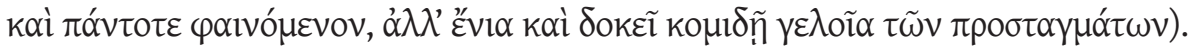
Plutarco avvalora questa affermazione con alcuni esempi storici relativi a leggi, usanze e prescrizioni, vigenti rispettivamente a Sparta (il divieto posto dagli efori di farsi crescere i baffi), a Roma (la manumissio vindicta e il testamentum per aes et libram) e ad Atene (la condanna prevista da Solone per chi, in una guerra civile, non

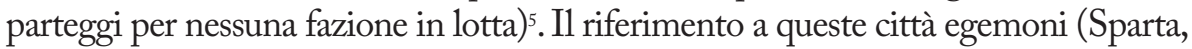
Roma e Atene) sembra assegnare un valore certo e universale a quanto affermato dal Cheronese, il quale al termine del capitolo riafferma, in una sorta di ringkomposition, come la stranezza (atopia) ${ }^{6}$ di tali provvedimenti sia soltanto apparente, da imputarsi interamente all'incapacità umana di cogliere le reali motivazioni dei legislatori.

\section{Arist. fr. 539 Rose: tre testimonianze plutarchee}

Tornando ai tre provvedimenti citati, appare evidente come Plutarco non li giudichi in realtà dei nomoi alogoi e/ a atopoi. Si consideri il primo $\pi \rho o ́ \sigma \tau \alpha \gamma \mu \alpha$, ossia il divieto emanato degli efori sui baffi, menzionato nel De sera e in altri due luoghi dell'opus plutarcheo:

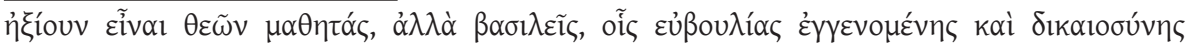

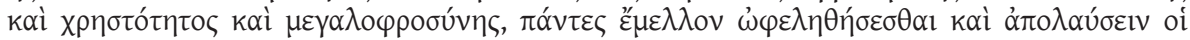

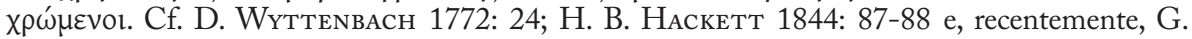
Ros Kam 2009: 91-93.

${ }^{4} \mathrm{Su}$ Minosse e altre figure di sovrani/ legislatori ispirati dalla divinità cf. Plu. Num. 4.11:

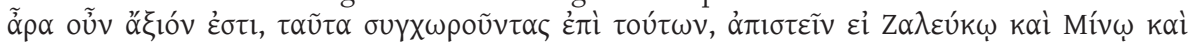

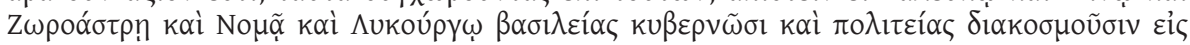

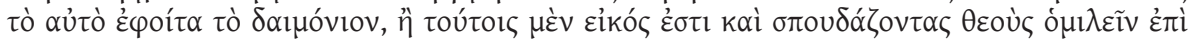

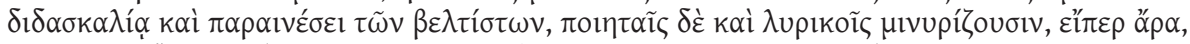

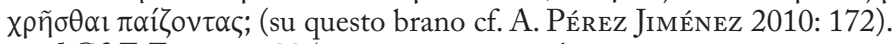

${ }^{5}$ Cf. F. Frazier: 225 in questo stesso volume.

${ }^{6}$ L' atopia è elemento ricorrente nei primi capitoli del De sera num. vind: essa caratterizza immediatamente l'anonimo epicureo, e ốto è ancora considerato Euripide, che nei suoi versi allude ad una Dike che insegue i colpevoli con passo lento

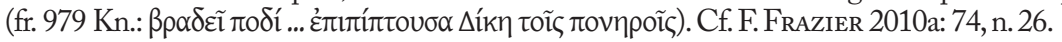


1) De sera num. vind. $4,550 \mathrm{~B}$

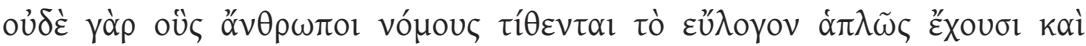

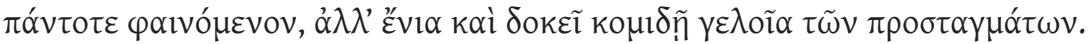

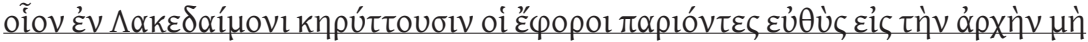

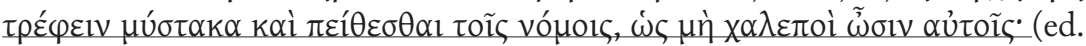
M. Pohlenz 1929: 399-400)7

\section{2) Cleom. 9.3-6}

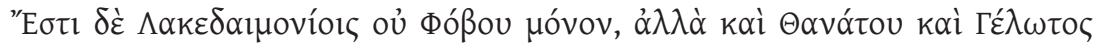

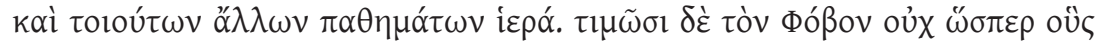

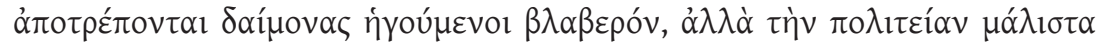

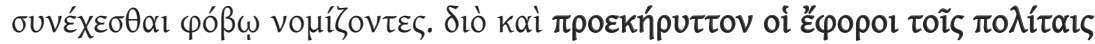

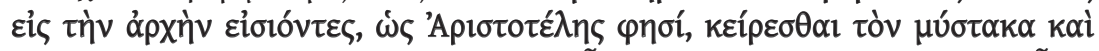

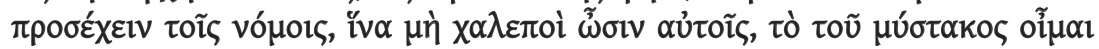

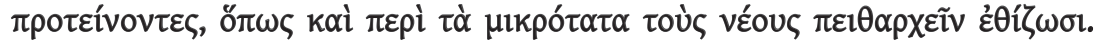

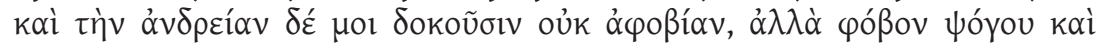

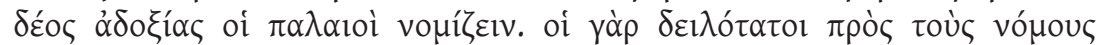

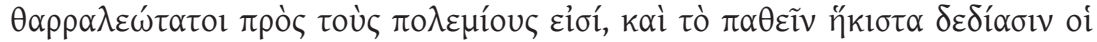

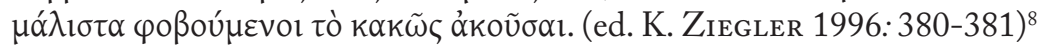

\section{3) Plu. fr. 90 Sandbach (= Schol. Hes. Op. 724-725)9

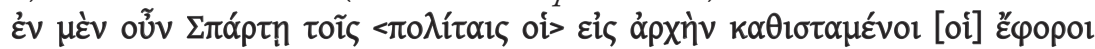

7 "D'altra parte, neppure le leggi che gli uomini si danno sono soggette a un solo criterio di razionalità universalmente manifesto, ma alcune norme sembrano francamente ridicole. $\mathbf{A}$ Sparta, per esempio, quando gli efori assumono la loro carica, proclamano che nessuno si lasci crescere i baffi, ed obbedisca alle leggi affinché queste non siano severe con loro" (trad. di G. Guidorizzi 1982: 132).

8 "Gli Spartani non hanno soltanto il tempio di Timore, ma anche quello della Morte, del Riso, di altri sentimenti del genere. Onorano il Timore non come quegli dei che si vogliono evitare perché ritenuti dannosi, ma perché credono che esso soprattutto tiene unito lo stato. $\mathbf{E}$ per questo che gli efori, entrando in carica, erano soliti ordinare ufficialmente ai cittadini, come dice Aristotele, di tagliarsi i baffi e rispettare le leggi perché esse non fossero aspre verso di loro, mettendo innanzi, io credo, la storia dei baffi per abituare i giovani a obbedire anche nelle minime cose. Per gli antichi il coraggio, mi pare, non consisteva nell'assenza di paura, ma nel timore del biasimo e nel terrore dell'infamia, I più timorosi della legge sono invero i più coraggiosi di fronte ai nemici; quelli che temono al massimo la mala fama temono pochissimo le sofferenze" (trad. di D. Magnino 1991: 193).

${ }^{9}$ Questo scolio fa parte dei cosiddetti scoli procliani, testimonianze di un perduto commentario agli Erga esiodei del neoplatonico Proclo. Questi si sarebbe a sua volta servito di un precedente commento plutarcheo: C. Faraggiana di Sarzana 1987: 22, scrive "Proclus s'est limité, à mon avis, à annoter le úró $\mu v \eta \mu \alpha$ de Plutarque dans les marges de son manuscrit. Il aurait, en d'autres termes, commenté un commentaire, par des observations critiques et par des intégrations". Lesistenza del commento plutarcheo, non menzionato nel Catalogo di Lampria, è confermata da Aulo Gellio in N.A. 20.8 (= fr. 102 Sandbach): Id etiam (...) multo mirandum est magis, quod apud Plutarchum in quarto in Hesiodum commentario legi (...). Sul rapporto tra i commenti esiodei di Plutarco e di Proclo cf., tra gli altri, A. Pertusi 1951:147-159; C. Faraggiana di Sarzana 1978: 17-40; T. Raiola 2010: 263-264, n. 1, con ampia bibliografia precedente. 


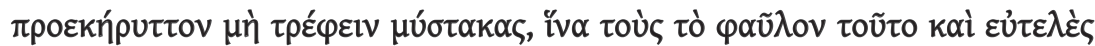

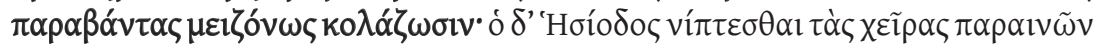

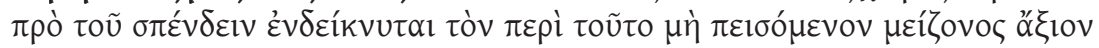

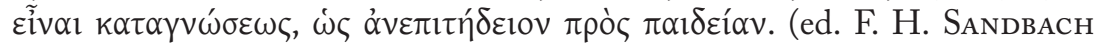
1967: 58 = F. H. SANDBACH 1969: 194) ${ }^{10}$

Dal confronto tra i tre testi (che costituiscono il fr. 539 Rose ${ }^{11}$ di Aristotele, citato esplicitamente da Plutarco solo nella Vita) si può immediatamente notare come la redazione del De sera 1) sia decisamente la più breve; 2) condivida con gli altri brani alcuni elementi fondamentali ${ }^{12}$, che possono essere così schematizzati:

a) il luogo in cui è emanato il $\pi \rho o ́ \sigma \tau \alpha \gamma \mu \alpha:$
De sera num.
Cleom.
fr. 90
ह̉v $\Lambda \alpha \kappa \varepsilon \delta \alpha i ́ p o v l$

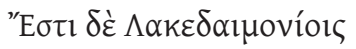

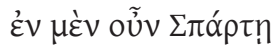

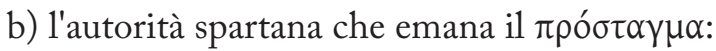

\begin{tabular}{|c|c|}
\hline 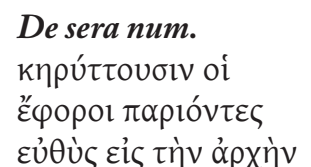 & 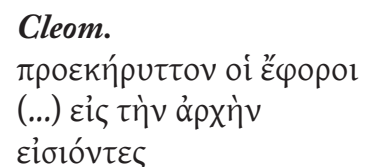 \\
\hline
\end{tabular}

De sera num.

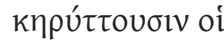

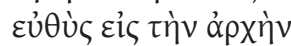

\section{fr. 90}

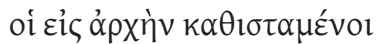

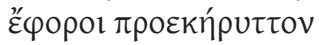

10 "A Sparta gli efori, nell'entrare in carica, proibivano per decreto ai cittadini la crescita dei baffi, col proposito di infliggere severe punizioni a chiunque trasgredisse un'imposizione tanto insignificante. Nell'esortare a lavarsi le mani prima della libagione, Esiodo vuole mostrare che chi è intenzionato a violare questa prescrizione merita una più severa reprimenda, essendo egli refrattario a ricevere un'educazione" (trad. M. De Simone, in P. Volpe 2010: 121).

${ }^{11}$ V. Rose 1886: 332-333.

${ }^{12}$ Un attento confronto tra i tre testi è proposto da B. W. Millis 1997: 575-577. In base alle somiglianze e differenze presenti nelle tre redazioni plutarchee della citazione aristotelica alcuni studiosi hanno congetturato su quale tipo di conoscenza (diretta o mediata da schede ed appunti) Plutarco abbia del testo di Aristotele: cf. G. Marasco 1978: 174, n. 25: "Una prova a sfavore dell'uso di schede e favorevole all'uso diretto potrebbe essere fornita dal ricorrere della stessa notizia sugli efori nel De sera numinis vindicta (550B), con notevoli differenze verbali, ed in un frammento ( 90 Sandbach), dove la prescrizione è spiegata in maniera diversa". Diversamente G. Marasco 1981: 426: "Queste differenze ed il fatto che nei due passi dei Moralia non è citato Aristotele, rafforzano la tesi che Plutarco, nel comporre la biografia di Cleomene, utilizzasse direttamente la $\Lambda \alpha \kappa \varepsilon \delta \alpha \iota \mu o v i ́ \omega v$ ro $\lambda ı \tau \varepsilon i ́ \alpha$ di Aristotele o schede tratte da essa in precedenza"; M. T. Schettino 1999: 649: "La coincidenza ad verbum tra il passo nella biografia di Agide e Cleomene e quello nel De sera numinis vindicta, benché in quest'ultimo non sia menzionato Aristotele, non sembra indicare una citazione a memoria ma tratta da un appunto scritto, che potrebbe derivare di prima mano dalla Lakedaimonion Politeia (...)”. Più in generale, sulla conoscenza dell'opera aristotelica da parte di Plutarco cf. F. H. SANDBAch 1982: 207-232; P. Donini 2004: 255-273; G. Karamanolis 2006: 85-126. 
c) il contenuto del $\pi \rho o ́ \sigma \tau \alpha \gamma \mu \alpha:$

\begin{tabular}{|c|c|c|}
\hline 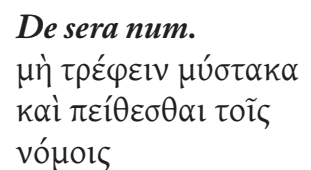 & 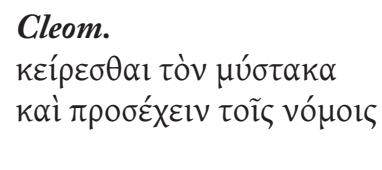 & 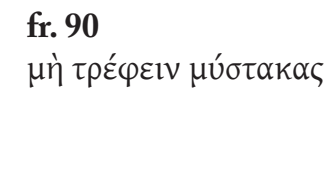 \\
\hline
\end{tabular}

d) l'eventuale sanzione per i trasgressori del $\pi \rho o ́ \sigma \tau \alpha \gamma \mu \alpha$ :
De sera num.
Cleom.
fr. 90

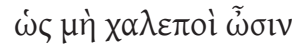

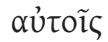

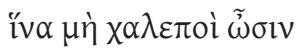

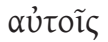

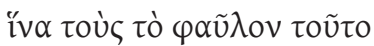

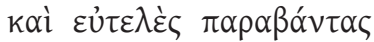

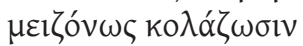

In particolare, quest'ultimo punto (d) richiede una riflessione di carattere esegetico: non appare chiaro, infatti, quale sia il soggetto della proposizione $\dot{\omega} \varsigma$

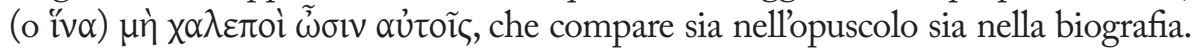
L'esegesi che sembrerebbe maggioritaria (direi canonica per il De sera ${ }^{13}$ ) ricava il soggetto della finale dal precedente toĩ lobbedienza alle leggi (anche a quelle che regolano aspetti di non grandissima importanza) farebbe sì che le stesse leggi non debbano essere più severe (cf. supra le traduzioni di Guidorizzi e Magnino). Due sembrano essere gli elementi a favore

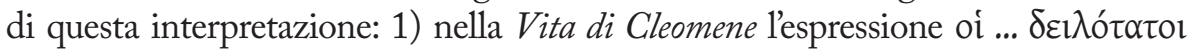

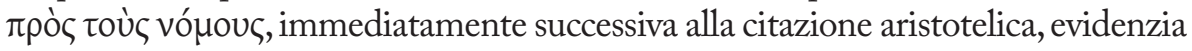

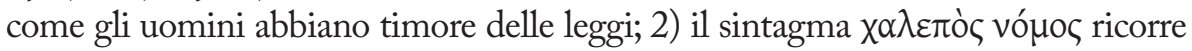
in Conv. sept. sap. 155F, dove è riferito ad una dura legge del saggio Pittaco ( $\Sigma \dot{\varepsilon} \gamma$ $\alpha^{\prime} \rho$,

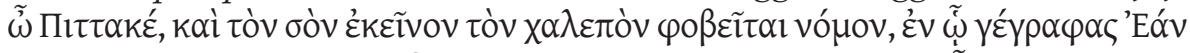

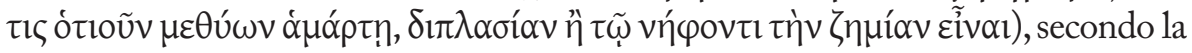
quale veniva raddoppiata la pena per i reati commessi in stato di ebbrezza.

${ }^{13}$ Oltre quella di Guidorizzi (cf. n. 7), cf. le traduzioni di A. O. Prickard 1918: 179 ("In Lacedaemon, for instance, the Ephors, when they first enter office, make proclamation that no one is to grow a moustache, and that men should obey the laws, that the laws may not be hard upon them"); Рh. DE LACY-B. Einarson 1959: 193 ("Thus in Lacedaemon, as soon as they take office, the ephors make a proclamation forbidding the wearing of moustaches and enjoining men to obey the laws, that the laws may not be harsh with them"); R. M. Aguilar 1996: 120121 ("Asi, en Lacedemonia, los éforos, en cuanto toman posesión de su magistratura, proclaman la prohibición de dejarse crecer los bigotes y la obediencia de las leyes, para que éstas no sean severas con ellos"); F. Frazier 2010b: 15 (“Ainsi à Lacédémone les éphores, dès leur entrée en charge, font proclamer l'obligation de ne pas se laisser pousser les moustaches et d'obéir aux

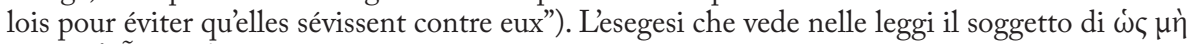

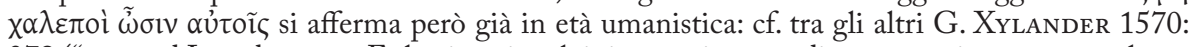
378 ("ut quod Lacedaemone Ephori statim ab inito magistratu edicunt ne quis mystacem alat et ut legibus obtemperent, ne eae ex ipsis sint difficiles observatu”) e J. Амүот 1572: 259 (“comme en Lacedemone les Ephores, aussi tost qu'ils sont instalez en leur magistrat, font publier à son de trompe, que personne ne porte moustaches, et que lon obeïsse volontairement aux loix, à fin qu'elles ne leur soient point dures"). 
Questa non è però la sola lettura possibile. In una nota al volgarizzamento del De Sera, opera dell'umanista Marcello Adriani, F. Ambrosoli scrive: "Questo medesimo riferisce Plutarco nella vita di Cleomene ove il G. Pompei (1816: 328329) tradusse con più verità: Quindiè che gli efori, quando entravano in magistratura, pubblicar faceano dal banditore a' lor cittadini, come dice Aristotele, che si radessero le basette, e che badasser bene alle leggi, acciocché non avesser eglino ad esser rigidi e severi con loro (...)" ${ }^{14}$. Secondo Pompei, che riferisce $\chi \alpha \lambda \varepsilon \pi$ oí agli efori stessi ${ }^{15}$, l'obbedienza alle leggi consentirebbe quindi agli Spartani di evitare la dura punizione degli efori, visti così nella loro funzione di custodi del nomos per conto della città. Un elemento a favore di questa seconda interpretazione potrebbe derivare dal confronto con il fr. 90 Sandbach. Nel commento ai versi degli Erga (a) non si fa menzione dei vó

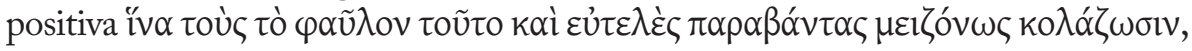
il cui soggetto sono appunto gli efori, che vietavano di portare i baffi con l'idea di punire con maggiore severità chi violasse questo divieto di poco conto e facile da osservare. Si può forse ipotizzare che il testo dello scolio contenga una sorta di parafrasi

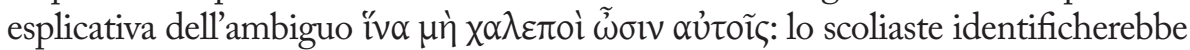
proprio negli efori coloro che sono preposti a punire con severità i trasgressori delle leggi. Questa prerogativa degli efori è inoltre ricordata da Senofonte nell'ottavo capitolo della Costituzione degli Spartani, capitolo dedicato appunto all'eforato e al ruolo svolto da questa magistratura nel fare di Sparta la città in cui maggiormente

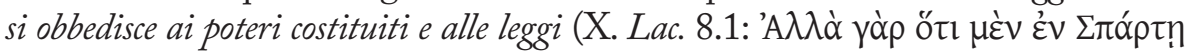

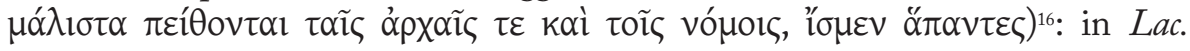
8.4 Senofonte afferma ancora che gli efori puniscono immediatamente chi notino

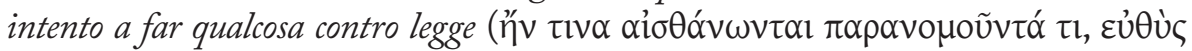

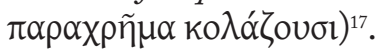

${ }^{14}$ M. Adriani 1827: 559; le traduzioni dell'Adriani furono pubblicate postume da F. Ambrosoli, cui è attribuibile il giudizio positivo sulla traduzione del Pompei.

${ }^{15}$ Simile esegesi, che vede negli efori il soggetto di $\chi \alpha \lambda \varepsilon \pi$ เi $\tilde{\omega} \sigma \mathrm{lv}$, si ritrova in altre traduzioni sempre relative al passo della Vita di Cleomene, e.g. in R. Flacelière 1976: 58 ("C'est pourquoi, quand les éphores entraient en charge, ils faisaient proclamer par le héraut... que les citoyens devaient se raser la moustache et se soumettre aux lois, pour qu'ils n'eussent pas à les punir"); $\mathrm{M}$. Nafissi 1991: 118 ("All'entrata in carica gli efori proclamavano ai cittadini ...di tagliarsi i baffi ed obbedire alle leggi, se non volevano che fossero severi con loro"; cf. inoltre M. NAFissi 2007: 340, che così interpreta Plu. Cleom. 9.3: "All'entrata in carica i "sorveglianti" annunciavano: "tagliatevi i baffi ed obbedite alla legge, se non volete incorrere nella nostra severità"'). Si noti inoltre che lo Xylander nel tradurre il passo della Vita di Cleomene si discosta dalla esegesi adottata per quello del De Sera (cf. supra n. 13), considerando gli efori soggetto anche della proposizione finale negativa: "Ideo etiam Ephori cum magistratum ineunt, civibus edicunt (...) ut superius labrum radant, ac legibus animum advertant, ne molesti ipsis esse cogantur" (G. XYlander 1580: 289).

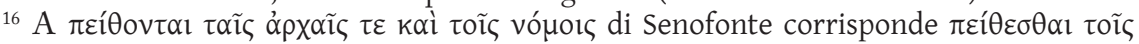
vópors di Plu. De sera num. vind. 550B. Sulle funzioni di controllo esercitate dagli efori a Sparta cf. inoltre L. Thommen 2003: 104.

${ }^{17}$ Sul passo di Senofonte cf. il commento di V. Gray 2007: 166-167. 


\section{Due motivi per una legge "illogica"}

Significative appaiono essere alcune divergenze che differenziano i tre brani plutarchei. In particolare, nella biografia di Cleomene, il Cheronese sembra offrire una 'duplice' spiegazione del proclama degli efori, 'bollato' invece nel De sera come apparentemente immotivato: 1) nel bios la citazione aristotelica è introdotta dalla congiunzione causale $\delta$ ió, che la vincola a quanto appena affermato da Plutarco, ossia che il phobos, la paura, onorata a Sparta come una divinità, sia il principale elemento di coesione della comunità lacedemone ( $\sigma v v \varepsilon ́ \chi \varepsilon \sigma \theta \alpha l)$. In tale ottica, radersi i baffi per timore di una punizione esemplare è testimonianza di come sia proprio il phobos a garantire l'obbedienza alle leggi da parte dei cittadini spartani; 2) è Plutarco a ritenere - oĩ $\alpha$ l sembra rimarcare il carattere soggettivo dell'ulteriore riflessione che segue la citazione di Aristotele - che il divieto degli efori possa svolgere inoltre una funzione paideutica, avendo come fine quello di educare

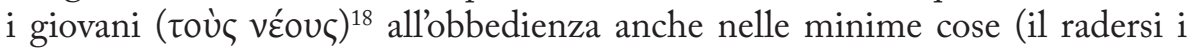
baffi), unobbedienza che poggia appunto sul già citato phobos, sul timore del

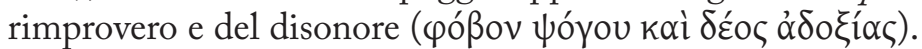

Soffermandosi inizialmente sul raffronto tra il brano della biografia e quello del De sera, è opportuno evidenziare come nell'opuscolo morale Plutarco passi sotto silenzio le ragioni del divieto spartano, esplicitate invece nella Vita di Cleomene. Tale reticenza è probabilmente da considerarsi il frutto di una scelta consapevole dell'autore: nel trattato, omettendo le spiegazioni relative al nomos stabilito dagli efori, il Cheronese può rimarcare maggiormente l'incapacità di alcuni uomini di comprendere la vera ragione di certi nomo ${ }^{19}$. L'aneddoto sui baffi degli Spartani può essere quindi impiegato come efficace esempio del modus operandi di Plutarco, che si serve della stessa citazione o dello stesso episodio storico in opere diverse, riadattandolo ai contesti diversi in cui si trova ad essere collocato ${ }^{20}$. Se nella Vita di Cleomene il divieto sui baffi è testimonianza efficace di come paura e rispetto per le leggi giovino alla città di Sparta, nel $D e$

${ }^{18}$ Per N. Richer 1998: 252-254, riveste grande importanza l'impiego da parte di Plutarco del sostantivo toù véous. In base alle testimonianze di Platone e, soprattutto, di Senofonte (P1. Lg. 6,760c e Xen. Mem. 1.2.35), a Sparta i neoi sarebbero da identificare con uomini di età inferiore ai trent'anni: essi dunque non avrebbero ancora terminato l'agoge e, ancora privi di una piena cittadinanza, non prenderebbero parte all'Apella.

${ }^{19} \mathrm{Cf}$. quanto afferma B. W. Millis 1997: 576-577, relativamente all'impiego di $\mu \eta \dot{~ \tau \rho \varepsilon ́ \varphi \varepsilon ı v ~}$

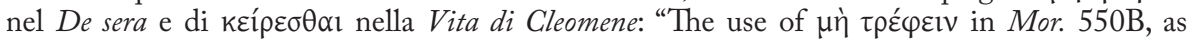

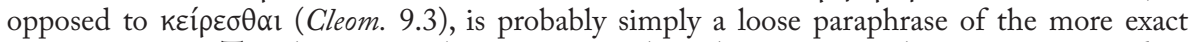
pronouncement. The change may be unintentional carelessness, since he is not interested in the Spartans per se in this passage, or could perhaps be deliberate, designed to highlight the apparent absurdity of the custom and thus strengthen his argument".

${ }^{20} \mathrm{Su}$ tale processo compositivo nelle opere del Cheronese cf. L. VAN DER STOckT 2004: 331-340; P. VOLPE 2007: 117-122. 
sera esso, opportunamente abbreviato e rifunzionalizzato, viene ricordato quale provvedimento apparentemente immotivato, e, quindi, atopos.

Venendo al fr. 90 Sandbach, la citazione della legge spartana è inserita all'interno del commento ai vv. 724-726 degli Erga, dove Esiodo riporta un particolare divieto di carattere religioso: Non offrire mai nell'ora dell'aurora a Zeus il nero vino con mani non pulite, né agli altri immortali. Lo scoliaste collega il precetto esiodeo di purezza rituale al divieto sui baffi decretato dagli efori mediante la

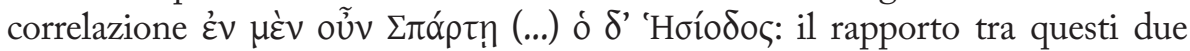
comandi (non libare con mani sudice / non lasciarsi crescere $i$ baffi) sembrerebbe

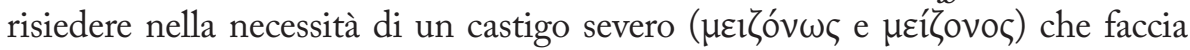
seguito alla mancata obbedienza alle norme ${ }^{21}$. L'impiego nel commento esiodeo

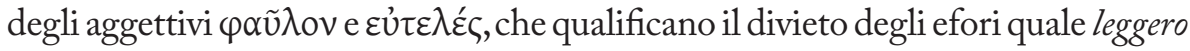
e di poco conto, e l'affermazione conclusiva sul rifiuto della paideia $a^{22}$ da parte di chi

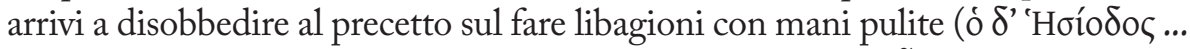

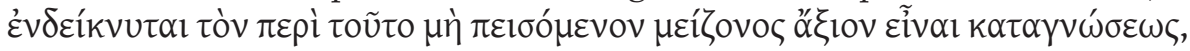

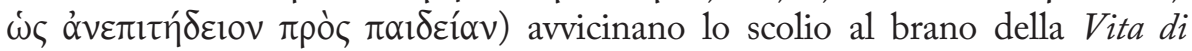
Cleomene: anche nella biografia Plutarco cataloga il comando sui baffi tra $\tau \grave{\alpha}$ $\mu \iota \kappa \rho o ́ \tau \alpha \tau \alpha$, le piccolissime prescrizioni alle quali bisogna comunque obbedire, sottolineandone al contempo la funzione paideutica, che consiste nell'educare i giovani a non trasgredire le norme stabilite dai magistrati, evitandone il castigo.

\section{I destinatari del divieto: problemi ecdotici nel fr. 90 Sandbach}

Un'ulteriore differenza tra $\mathrm{i}$ tre testimoni plutarchei del frammento aristotelico 539 Rose è rappresentata dall'indicazione dei destinatari del proclama degli efori, un elemento che porta con sé alcune difficoltà di carattere sia testuale sia interpretativo. Nella forma riassunta del De sera, infatti, Plutarco impiega il

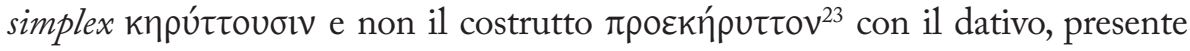
sia nel bios sia nel fr. 90. Nell'opuscolo non viene quindi precisato a chi sia rivolto l'editto dei magistrati spartani: un'omissione, quella dei destinatari, che crea un ulteriore dubbio nel determinare a chi vada riferito il successivo pronome $\alpha$ Ủंoĩ

${ }^{21}$ Cf. il commento allo scolio di P. Marzillo 2010: 359.

${ }^{22} \mathrm{Il}$ contenuto paideutico dello scolio ben si conforma agli interessi di Proclo: C. FAraggiana di SARZANA 1978: 22, sottolinea come i riferimenti negli 'scoli procliani' a determinati aneddoti storici siano impiegati quali "esempio e conferma di un precetto o di una interpretazione etico-pedagogica".

${ }^{23}$ B.W. Millis 1997: 576 evidenzia inoltre come l'impiego da parte di Plutarco dell'imperfetto

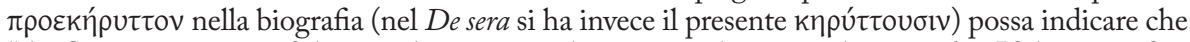
"the Spartan practice of shaving their moustaches was not known in his own day. If the imperfect was Aristotle's, it is possible that it was not a regular practice in his time either, and accordingly it is worth noting that no other reference to the custom exists in the classical period". Cf. inoltre G. Marasco 1981: 427: "In ogni modo, le stesse contraddizioni in cui cade Plutarco nello spiegare i motivi dell'usanza testimoniano che, già ai suoi tempi, i motivi di essa non erano più chiari”. 
della proposizione finale negativa. Le numerose traduzioni del De sera, da quelle umanistiche alle più recenti, appaiono tuttavia concordi (a) nell'individuare nei

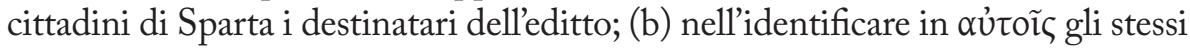
Spartani $^{24}$. Così interpretata, la testimonianza del De sera viene a coincidere

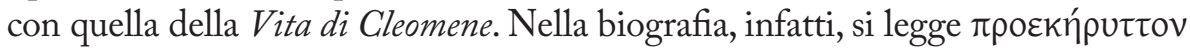
oi

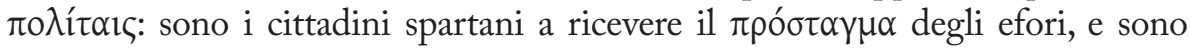
sempre gli stessi cittadini a essere puniti in caso di disobbedienza alle leggi.

Maggiori problemi presenta invece il fr. 90, che fin dalle prime edizioni a stampa è stato fatto oggetto di numerosi interventi, di cui vorrei, in conclusione, valutare opportunità ed efficacia. D. WyтtenвAсн 1800: 797 stampa la porzione di frammento inerente al nomos degli efori, riproducendo prudentemente la paradosi dei manoscritti, ossia

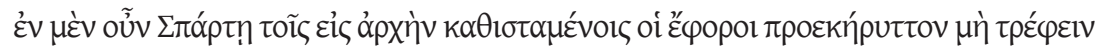

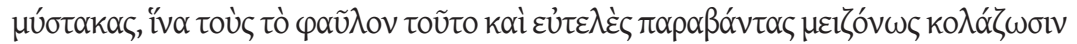

e traduce

Et Spartae quidem Ephori magistratibus, simulatque constituti essent, edicebant ne mystaces, id est barbas infra nares, alerent; ut eos qui leve hoc ac tenue praeceptum neglexissent, gravius punirent ${ }^{25}$.

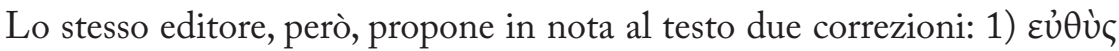

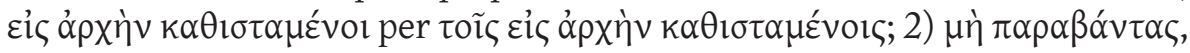

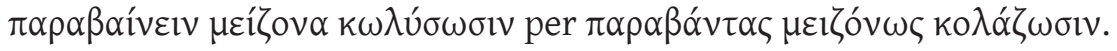

Il primo intervento è menzionato da Wyttenbach già nelle animadversiones che corredano la sua editio singularis del De sera ${ }^{26}$ : nella stessa sede lo studioso ricorda un'altra proposta di D. Ruhnken, il quale suggerisce a sua volta di

${ }^{24}$ Più esplicite a tal proposito appaiono le traduzioni di A.P. Peabody 1885: 9 ("Thus, in Lacedaemon, the Ephori when they come into office make immediate proclamation that no man shall let the beard on the upper lip grow, and that the laws must be so obeyed that they shall never be annoying to the citizens") e di M. Hose 2002: 47 ("In Sparta verkündeten die Ephoren bei Amtsantritt, man solle sich keinen Schnurrbart wachsen lassen und den Gesetzen gehorchen, damit sie ihnen [den Spartiaten] nicht beschwerlich werden".

${ }^{25}$ Cf. la traduzione italiana in D. BAвUт 2003: 545: "A Sparta gli efori vietavano ai magistrati entranti in carica di portare i baffi, con l'idea di punire severamente chi trasgredisse questa prescrizione insignificante e facile da osservare".

${ }^{26}$ Nella nota di commento di D. Wyttenbach 1772: 25 (= D. Wyttenbach 1821: 333), lo scolio procliano viene citato dall'edizione esiodea di D. Heinsius, pubblicata a Leida 1603; nel

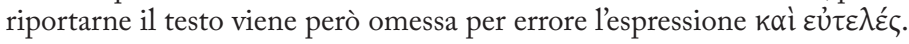




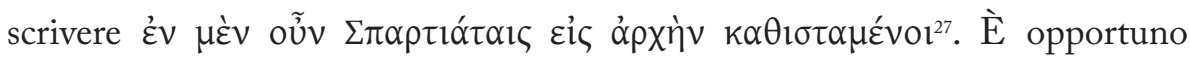
evidenziare come queste correzioni sembrino nascere dal confronto tra il testo del fr. 90 e quelli degli altri due brani plutarchei: se l'avverbio عủ $\theta u ́ c$ compare nel

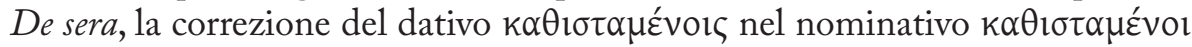
(concordato così con il soggetto oi $\tilde{\varepsilon} \varphi$ opol) appare derivata dalla presenza, sia

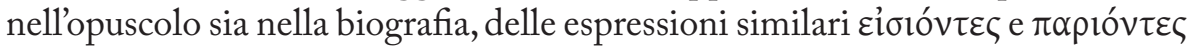

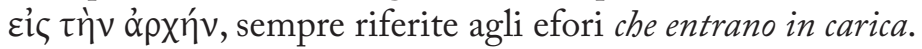

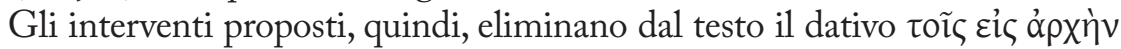

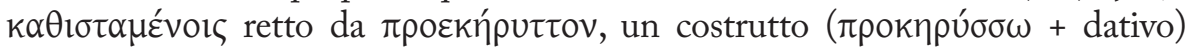
presente, come sopra ricordato, anche nella Vita di Cleomene: gli studiosi non ritengono possibile che destinatari del comando eforale siano alcuni Spartani chiamati a ricoprire qualche altra magistratura o incarico (cf. magistratibus della traduzione di Wyttenbach) sotto il controllo degli efori. Tuttavia, di un possibile potere di sorveglianza dell'eforato su altri magistrati si ha testimonianza nel già

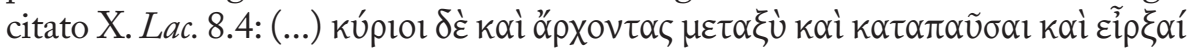

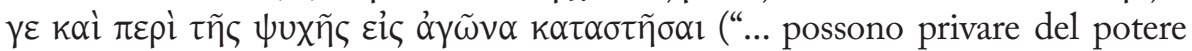
persone che sono ancora in carica per un compito istituzionale, imprigionarle e costringerle a mettere in gioco la loro vita in un processo").

Il 'processo', iniziato da Wyttenbach/ Ruhnken, di assimilazione del testo del commentario esiodeo alle altre due testimonianze plutarchee, che prevede l'eliminazione per via congetturale delle divergenze tra i tre brani, continua nei secoli successivi. Se Gaisford, Dübner, Rose e Bernardakis mantengono anch'essi la paradosi dei manoscritti ${ }^{28}$, A. Pertusi 1955 I: 222, nell'edizione degli scholia vetera agli Erga esiodei, stampa

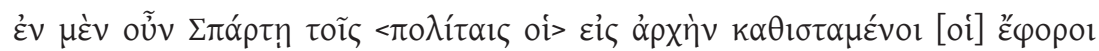

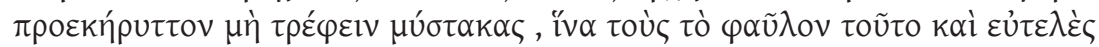

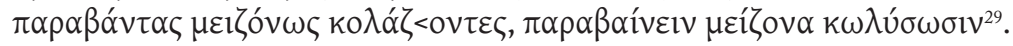

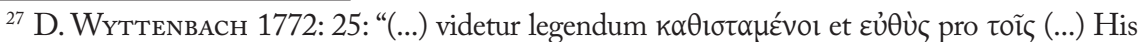
ita in chartam conjectis, eximiam mecum istam animadversionem communicavit Cel. Ruhnkenius:

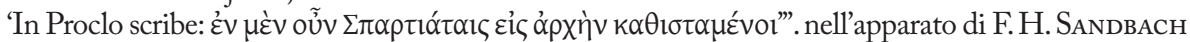

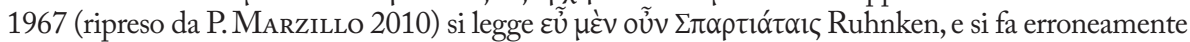
supporre che Ruhnken individuasse negli Spartiati i destinatari della legge eforale ( $\Sigma \pi \alpha \rho \tau$ ́ó $\tau \alpha \iota \varsigma$

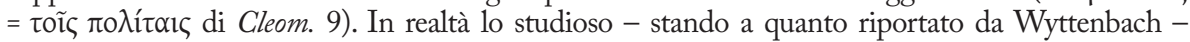

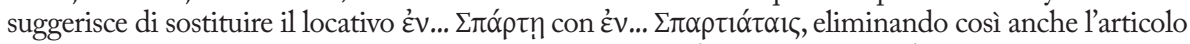

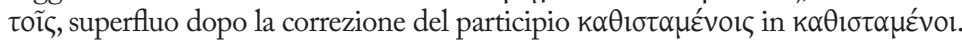

${ }_{28}$ Th. Gaisford 1820: 323; F. DüBner 1855: 30 (che ristampa anche la traduzione di Wyttenbach); V. Rose 1886: 332-333; G. N. Bernardakis 1896: 88-89 (= fr. 72).

29 "A Sparta dunque gli efori, assumendo la loro funzione, ingiungevano preventivamente ai cittadini di non farsi crescere i baffi, affinché, punendo piuttosto severamente i trasgressori di questa banale e insignificante prescrizione, si impedissero maggiori trasgressioni” (trad. di C. CASSANMAGNAGo 2009: 843). Il testo stabilito da Pertusi è accolto da O. Gigon 1987: 668 (fr. 545.3). 


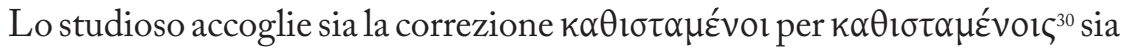

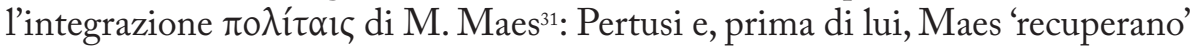

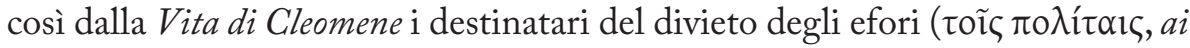
cittadini), dopo che Wyttenbach (con il suo maestro Ruhnken) ha 'eliminato' il

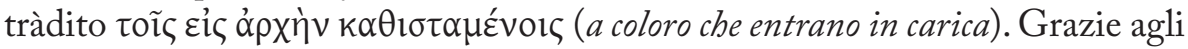
interventi di Pertusi, accolti più recentemente anche da Sandbach (cf. supra) e P. Marzillo 2010: 254, l'inizio del frammento viene a coincidere con il testo della biografia, salvo alcune minime differenze:

\section{fr. 90}

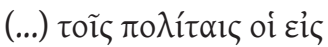

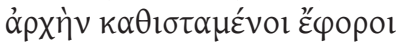

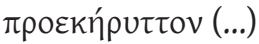

\section{Cleom. 9}

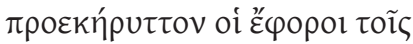

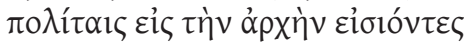

(...)

Al termine di questo breve status quaestionis, resta da interrogarsi sull'opportunità o meno di intervenire sul testo del frammento, che offre un senso accettabile (cf. supra le traduzione dello stesso Wyttenbach e di Babut), al solo fine di uniformarne forma e significato alla testimonianza offerta dalla biografia di Cleomene, ritenuta forse più completa e attendibile. In realtà, una simile operazione sembrerebbe sconsigliabile, data la natura stessa del testo in cui il frammento plutarcheo è collocato, ossia uno scolio assemblato con materiali di varia provenienza e sottoposto nel corso della tradizione a revisione da parte di più redattori. È ipotizzabile che lo scoliaste, oramai ignaro degli originali nomoi spartani, possa aver infine trasmesso una versione diversa (e probabilmente errata) della testimonianza relativa all'editto spartano sui baffi, ma ciò non sembra comunque autorizzare gli editori moderni ad intervenire per ricostruire anche nel frammento plutarcheo quella che essi ritengono la versione canonica ${ }^{32}$, considerato che anche le testimonianze propriamente plutarchee del De sera e della Vita di Cleomene presentano - come si è cercato di dimostrare - alcune specificità e differenze non sempre trascurabili.

${ }^{30}$ L'editore accoglie parzialmente anche la correzione proposta da Wyttenbach per la seconda proposizione del passo (cf. supra): " $\pi \alpha \rho \alpha \beta \alpha i ́ v \varepsilon ı v ~-~ \kappa \omega \lambda u ́ \sigma \omega \sigma ı v ~ p r a e e u n t e ~ W y t t e n b a c h$ correxi” così annota A. Pertusi 1955 II: 196.

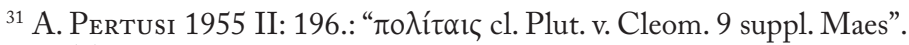

${ }^{32}$ Cf. T. Raiola 2010: 274-275 a proposito del frammento plutarcheo 81 Sandbach, sempre appartenente al commentario esiodeo. 


\section{Abbreviazioni bibliografiche}

Adriani,M.,Opuscolidi Plutarcovolgarizzati daMarcello Adriani,nuovamente confrontati col testo e illustrati con note da Francesco Ambrosoli, III, Milano, 1827.

Aguilar, R.M.,Plutarco, Obras moralesydecostumbres (Moralia), introducciones, traducciones y notas por Rosa María Aguilar, VIII, Madrid, 1996.

Амуот, J., Les cuvres morales et meslées de Plutarque, translatées du grec en françois, par Messire Jacques Amyot, Paris, 1572.

Babut, D., Plutarco e lo stoicismo, edizione italiana a cura di A. Bellanti, Milano, 2003 (titolo originale: Plutarque et le stoïcisme, Paris, 1969).

Bernardakis, G. N., Plutarchi Chaeronensis Moralia recognovit G.N. Bernardakis, VII (Plutarchi Fragmenta vera et spuria), Lipsiae, 1896.

Cassanmagnago, C., Esiodo, Tutte le Opere e i Frammenti con la prima traduzione degli scolii, a cura di C. Cassanmagnago, Milano, 2009.

de Lacy Ph.; Einarson, B., Plutarch's Moralia, with an English Translation by Ph. De Lacy and B. Einarson, VII, London-Cambridge, 1959.

Donini, P., "Plutarco e Aristotele", in I. Gallo (ed.), La biblioteca di Plutarco, Napoli, 2004: 255-274.

Dübner, F., Plutarchi Fragmenta et Spuria cum codicibus contulit et emendavit Fr. Dübner, Parisiis, 1855.

Faraggiana di Sarzana, C., "Il commentario procliano alle Opere e i Giorni”, Aevum, 52, 1978: 17-40.

Faraggiana di Sarzana, C., "Le Commentaire à Hésiode et la 'paideia' encyclopédique de Proclus", in J. Pépin-H.D. Saffrey (eds.), Proclus lecteur et interprète des anciens, Paris, 1987: 21-41.

Flacelière, R., Plutarque, Vies, texte établi et traduit par R. Flacelière et E. Chambry, XI (Agis-Cléomène; Les Gracques), Paris, 1976.

Frazier, F., "Le De sera, dialogue pythique: Hasard et Providence, Philosophie et Religion dans la pensée de Plutarque", in F. Frazier ; D. F. Leão (eds.), Tychè et Pronoia. La marche du monde selon Plutarque, Coimbra, 2010a.

Frazier, F., Plutarque, Sur les délais de la justice divine. texte établi par Yvonne Vernière. Traduction, introduction et notes par F. Frazier, Paris, 2010b.

Gaisford, Th., Poetae minores graeci, praecipua lectionis varietate et indicibus instruxit Th. Gaisford, III, Oxonii, 1820.

Gigon, O., Aristotelis Opera. III, librorum deperditorum fragmenta collegit et annotationibus instruxit O. Gigon, Berlin-New York, 1987.

Gray, V., Xenophon on Government, Cambridge, 2007. 
Guidorizzi, G., Plutarco. Il demone di Socrate (trad. e note di A. Aloni), Iritardi della punizione divina (trad. e note di G. Guidorizzi), introduzione e nota informativa di D. Del Corno, Milano, 1982.

Hackett, H. B., Plutarch on the Delay of the Deity in the Punishment of the Wicked, with notes, by H.B. Hackett, New York, 1844.

Hose, M., Aristoteles, Die historischen Fragmente. Übersetzt und erläutert von M. Hose, (Aristoteles, Werke in deutscher Übersetzung; 20/111), Berlin, 2002.

Karamanolis, G., Plato and Aristotle in Agreement? Platonists on Aristotle from Antiochus to Porphyry, Oxford, 2006.

Magnino, D., Plutarco, Agide e Cleomene, Tiberio e Caio Gracco, introduzione, traduzione e note di D. Magnino, con contributi di B. Scardigli e M. Manfredini, Milano, 1991.

Marasco, G., "Aristotele come fonte di Plutarco nelle biografie di Agide e Cleomene", Athenaeum, 56, 1978: 170-181.

Marasco, G., Commento alle biografie plutarchee di Agide e di Cleomene, Roma, 1981.

Marzillo, P., Der Kommentar des Proklos zu Hesiods 'Werken und Tagen' Edition, Übersetzung und Erläuterung der Fragmente (Classica Monacensia Bd. 33), Tübingen, 2010.

Millis, B.W., "Antiphanes fr.46 K-A and the Problem of Spartan Moustaches", CQ, 47, 1997: 574-578.

NAfissi, M., La nascita del kosmos. Studi sulla storia e la società di Sparta, Perugia, 1991.

Nafissi, M., "Forme di controllo a Sparta", Il pensiero politico, 40, 2007: 329344.

Peabody, A. P., Plutarch on the Delay of the Divine Justice, translated, with an introduction and notes, by A.P. Peabody, Boston, Mass., 1885.

Pérez Jiménez, A., "La Providencia como salvaguarda de los proyectos históricos bumanos en las Vidas paralelas", in F. Frazier; D.F. Leão (eds.), Tychè et Pronoia. La marche du monde selon Plutarque, Coimbra, 2010: 169-181.

Pertusi, A., "Intorno alla tradizione manoscritta degli scolii di Proclo ad Esiodo. IV", Aevum, 25, 1951: 147-159.

Pertusi, A., Scholia vetera in Hesiodi Opera et Dies, recensuit A. Pertusi,Milano, 1955.

Pohlenz, M., Plutarchi Moralia, recensuerunt et emendaverunt W.R. Paton-M. Pohlenz-W. Sieveking, III, Lipsiae, 1929.

Pompei, G., Vite degli uomini illustri di Plutarco, volgarizzate da Girolamo Pompei, VII, Milano, 1816. 
Prickard, A.O., Selected Essays of Plutarch. Vol.II.Translated with Introduction by A. O. Prickard, Oxford, 1918.

Raiola, T., "Plutarco e non Plutarco. Sul frammento 81 Sandbach", in G. Zanetto; S. Martinelli Tempesta (eds.), Plutarco: lingua e testo (Quaderni di Acme 122), Milano, 2010: 263-279.

Richer, N., Les ephores. Etudes sur l'histoire et sur l'image de Sparte (VIII ${ }^{e}-I I^{e}$ siècles avant Jésus- Christ), Paris, 1998.

Rose, V., Aristotelis qui ferebantur librorum deperditorum fragmenta, collegit V. Rose, Leipzig, 1886.

Rosкam, G., Plutarch's Maxime cum principibus philosopho esse disserendum. An Interpretation with Commentary, Leuven, 2009.

Sandbach, F. H., "Plutarch and Aristotle", ICS, 7, 1982: 207-232.

Sandbach, F. H., Plutarchi Moralia, edidit F. H. Sandbach, VII, Lipsiae, 1967.

SAndbach, F. H., Plutarch's Moralia, XV (Plutarch's Fragments), London, 1969.

Schettino, M.T., "Le politeiai aristoteliche nel corpus plutarcheo", in A. Perez Jimenez; J. García López; R. M. Aguilar (eds.), Plutarco, Pláton y Aristoteles, Madrid, 1999: 643-655.

Thommen, L., Sparta. Verfassungs- und Sozialgeschichte einer griechischen Polis, Stuttgart-Weimar, 2003.

VAn Der Stockt,L., "Plutarch in Plutarch: the Problem of the Hypomnemata", in I. Gallo (ed.), La Biblioteca di Plutarco, Napoli, 2004: 331-340.

Volpe, P., "Plutarchus in Plutarcho: de primo frigido e quaest. conv. VI, 4-5", Ploutarchos, 4, 2007: 117-122.

Volpe, P., Plutarco, Frammenti, a cura di P. Volpe Cacciatore, Napoli, 2010.

Wyttenbach, D., Plutarchi liber de sera numinis vindicta, recensuit, emendavit, illustravit D. Wyttenbach, Lugduni Batavorum, 1772.

Wyttenbach, D., Plutarchi Chaeronensis Moralia, id est opera, exceptis Vitis, reliqua. Graeca emendavit, notationem emendationum, et Latinam Xylandri interpretationem castigatam, subjunxit, animadversiones explicandis rebus ac verbis, item indices copiosos, adjecit D. Wyttenbach, V 2, Oxonii, 1800.

Wyttenbach, D., Danielis Wyttenbachii animadversiones in Plutarchi Moralia, Oxonii, 1821.

Xylander, G., Plutarchi Chaeronensis Moralia, quae usurpantur [...] G. Xylandro Augustano interprete, Basileae, 1570.

Xylander, G., Plutarchi Chaeronensis, ... Parallela, id est, Vitae illustrium virorum Graecorum et Romanorum, [...] Gulielmo Xylandro Augustano 
Un nomos atopos? Gli Efori e i baffi degli Spartani.

interprete. [...] Cum indice rerum et verborum copioso, Francoforti ad Moenum, 1580.

Ziegler, K., Plutarchi Vitae parallelae, iterum recensuit $K$. Ziegler, editionem correctiorem cum addendis curavit H.Gärtner, III 1, Stuttgart-Leipzig, 1996. 